\title{
Optimization of GaAs Amplification Photodetectors for $700 \%$ Quantum Efficiency
}

\author{
Joachim Piprek, Senior Member, IEEE, Daniel Lasaosa, Donato Pasquariello, Associate Member, IEEE, and \\ John E. Bowers, Fellow, IEEE
}

\begin{abstract}
We investigate the device physics of novel GaAs waveguide photodetectors with integrated photon multiplication. Such detectors have the potential to achieve simultaneously high saturation power, high speed, high responsivity, and quantum efficiencies above $100 \%$. Our device design vertically combines a bulk photodetector ridge waveguide region with laterally confined quantum wells for amplification. Measurements on the first device generation show quantum efficiencies of only 56\%. Advanced device simulation is employed to analyze these devices and to reveal performance limitations. Excellent agreement between simulations and measurements is obtained. Device design optimization is proposed, promising more than $\mathbf{7 0 0 \%}$ efficiency.
\end{abstract}

Index Terms-Integrated photonics, numerical simulation, optoelectronics, photodetector, semiconductor optical amplifier, waveguide, III-V semiconductor devices.

\section{INTRODUCTION}

$\mathbf{I}$ $\mathrm{N}$ WAVEGUIDE photodetectors, photon flux and carrier transport are perpendicular to each other, which enables high-data-rate applications, since thin absorption layers allow for short transit times of the generated carriers. The detection efficiency is increased by using long waveguides. Ideally, every photon generates one electron-hole pair giving $100 \%$ quantum efficiency. However, only a fraction of the optical signal is usually converted into an electrical signal. To obtain stronger electrical output power, the optical input power may be enhanced by preamplification, but this method is limited by saturation effects. In order to overcome this problem, we have recently proposed a monolithic integration of detector and amplifier, called traveling-wave amplification photodetector (TAP detector), which simultaneously amplifies and absorbs the incoming light, promising quantum efficiencies well above $100 \%$. [1] We have previously evaluated the microwave characteristics, the signal modulation bandwidth, and the noise figure of TAP detectors. [2] In this paper, we analyze the detection efficiency for our most recent GaAs TAP detector fabricated, which features the vertical coupling of amplifier and detector region as well as oxide confinement layers (Fig. 1). The quantum efficiency measured with this device is still below $100 \%$. Using advanced device simulation, we here reveal the limiting physical mechanisms and we propose an optimized device design for high quantum efficiency.

Manuscript received December 16, 2002; revised May 16, 2003.

The authors are with the Electrical and Computer Engineering Department, University of California, Santa Barbara, CA 93106-9560 USA (e-mail: piprek@ece.ucsb.edu).

Digital Object Identifier 10.1109/JSTQE.2003.820405

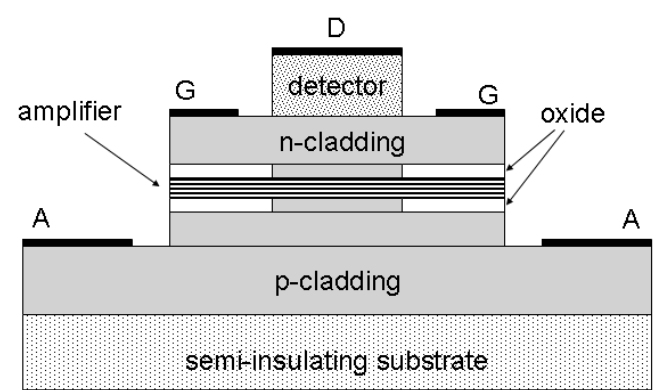

Fig. 1. Cross section of the TAP detector (D-detector contact; G-ground contact; A-amplifier contact). The detector width and the oxide aperture are $W=3 \mu \mathrm{m}$.

The schematic cross section of our three-terminal device is shown in Fig. 1 and the layer structure is listed in Table I. The GaAs ridge-waveguide detector diode is grown on top of a multiquantum well (MQW) amplifier diode. Fig. 2 pictures the facet of a fabricated device. The incoming optical wave stimulates the generation of additional photons within the amplification layers. Some of these new photons are absorbed in the detection layer, thus increasing the photocurrent. Higher pump current in the amplifier diode leads to higher photocurrent in the detector diode. With sufficient amplifier gain, the number of the detected photons can be larger than the number of incoming photons, giving more than $100 \%$ quantum efficiency. Optical and electrical confinement to the center of the device is provided by the ridge-waveguide structure as well as by lateral oxidation. The oxide aperture is the same as the ridge width of $W=3 \mu \mathrm{m}$. The device length is $L=300 \mu \mathrm{m}$, unless noted otherwise.

In the following, we analyze the steady-state device physics of this TAP detector using advanced numerical simulation. The commercial software packages APSYS [3] and BeamPROP [4] are partially employed. We consider low light power here so that the material properties of our waveguide are uniform in longitudinal $z$ direction. Measurements are used to validate the accuracy of the simulation.

\section{Current Flow}

The two-dimensional (2-D) flow of electrons and holes is simulated based on the common semiconductor transport equation [5]. The drift-diffusion model includes Fermi statistics of carriers and thermionic emission at hetero-interfaces. Doping densities and carrier mobilities of our device are listed in Table I [3], [6]. 
TABLE I

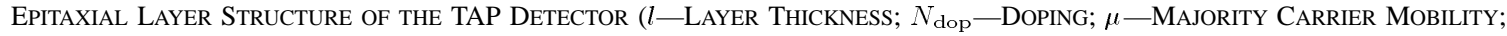
$\kappa$-Thermal CONDUCTIVITY (OXIDE: $0.1 \mathrm{~W} / \mathrm{cmK}$ ), $n_{r}$-REFRACTIVE INDEX (OXIDE: 1.7 ), AT RoOM TEMPERATURE). INTRINSIC (i) LAYERS ARE ASSUMED TO EXHIBIT LOW p-TYPE BACKGROUND DOPING

\begin{tabular}{|c|c|c|c|c|c|}
\hline Parameter & $l$ & $N_{\text {dop }}$ & $\mu$ & $\kappa$ & $n_{r}$ \\
\hline Unit & $\mathrm{nm}$ & $1 / \mathrm{cm}^{3}$ & $\mathrm{~cm}^{2} / \mathrm{Vs}$ & $\mathrm{W} / \mathrm{cmK}$ & - \\
\hline i-GaAs (absorption) & 300 & $1 \times 10^{16}$ & 290 & 0.44 & 3.65 \\
\hline $\mathrm{n}-\mathrm{Al}_{0.15} \mathrm{Ga}_{0.85} \mathrm{As}$ (cladding) & 100 & $5 \times 10^{18}$ & 1000 & 0.22 & 3.54 \\
\hline $\mathrm{n}-\mathrm{Al}_{0.2} \mathrm{Ga}_{0.8} \mathrm{As}$ (cladding) & 300 & $1 \times 10^{18}$ & 1300 & 0.17 & 3.50 \\
\hline $\mathrm{n}-\mathrm{Al}_{x} \mathrm{Ga}_{1-x} \mathrm{As}(\mathrm{x}=0.9$ to 0.2$)$ & 16.5 & $1 \times 10^{18}$ & graded & 0.10 & graded \\
\hline $\mathrm{n}-\mathrm{Al}_{0.9} \mathrm{Ga}_{0.1} \mathrm{As}$ (oxidation) & 6 & $1 \times 10^{18}$ & 60 & 0.10 & 2.99 \\
\hline $\mathrm{n}-\mathrm{Al}_{0.98} \mathrm{Ga}_{0.02} \mathrm{As}$ (oxidation) & 32 & $1 \times 10^{18}$ & 65 & 0.86 & 2.94 \\
\hline $\mathrm{n}-\mathrm{Al}_{0.9} \mathrm{Ga}_{0.1} \mathrm{As}$ (oxidation) & 6 & $1 \times 10^{18}$ & 60 & 0.10 & 2.99 \\
\hline $\mathrm{n}-\mathrm{Al}_{x} \mathrm{Ga}_{1-x} \mathrm{As}(\mathrm{x}=0.15$ to 0.9$)$ & 18.2 & $1 \times 10^{18}$ & graded & 0.10 & graded \\
\hline $\mathrm{n}-\mathrm{Al}_{0.15} \mathrm{Ga}_{0.85} \mathrm{As}$ (waveguide) & 90 & $1 \times 10^{18}$ & 1800 & 0.22 & 3.54 \\
\hline i- $\mathrm{Al}_{0.15} \mathrm{Ga}_{0.85} \mathrm{As}$ (waveguide) & 10 & $1 \times 10^{16}$ & 150 & 0.22 & 3.54 \\
\hline i-GaAs (quantum well) & 8 & $1 \times 10^{16}$ & 310 & 0.05 & 3.65 \\
\hline $\mathrm{i}-\mathrm{Al}_{0.15} \mathrm{Ga}_{0.85} \mathrm{As}$ (barrier) & 8 & $1 \times 10^{16}$ & 260 & 0.05 & 3.54 \\
\hline i-GaAs (quantum well) & 8 & $1 \times 10^{16}$ & 310 & 0.05 & 3.65 \\
\hline $\mathrm{i}-\mathrm{Al}_{0.15} \mathrm{Ga}_{0.85} \mathrm{As}$ (barrier) & 8 & $1 \times 10^{16}$ & 260 & 0.05 & 3.54 \\
\hline i-GaAs (quantum well) & 8 & $1 \times 10^{16}$ & 310 & 0.05 & 3.65 \\
\hline $\mathrm{i}-\mathrm{Al}_{0.15} \mathrm{Ga}_{0.85} \mathrm{As}$ (barrier) & 8 & $1 \times 10^{16}$ & 260 & 0.05 & 3.54 \\
\hline i-GaAs (quantum well) & 8 & $1 \times 10^{16}$ & 310 & 0.05 & 3.65 \\
\hline i- $-\mathrm{Al}_{0.15} \mathrm{Ga}_{0.85} \mathrm{As}$ (waveguide) & 60 & $1 \times 10^{16}$ & 260 & 0.22 & 3.54 \\
\hline $\mathrm{p}-\mathrm{Al}_{0.2} \mathrm{Ga}_{0.8} \mathrm{As}$ (waveguide) & 20 & $5 \times 10^{17}$ & 135 & 0.17 & 3.50 \\
\hline $\mathrm{p}-\mathrm{Al}_{x} \mathrm{Ga}_{1-x} \mathrm{As}(\mathrm{x}=0.9$ to 0.2$)$ & 18.2 & $1 \times 10^{18}$ & graded & 0.10 & graded \\
\hline $\mathrm{p}-\mathrm{Al}_{0.9} \mathrm{Ga}_{0.1} \mathrm{As}$ (oxidation) & 6 & $3 \times 10^{17}$ & 60 & 0.10 & 2.99 \\
\hline $\mathrm{p}-\mathrm{Al}_{0.98} \mathrm{Ga}_{0.02} \mathrm{As}$ (oxidation) & 32 & $3 \times 10^{17}$ & 60 & 0.86 & 2.94 \\
\hline $\mathrm{p}-\mathrm{Al}_{0.9} \mathrm{Ga}_{0.1} \mathrm{As}$ (oxidation) & 6 & $3 \times 10^{17}$ & 60 & 0.10 & 2.99 \\
\hline $\mathrm{p}-\mathrm{Al}_{x} \mathrm{Ga}_{1-x} \mathrm{As}(\mathrm{x}=0.2$ to 0.9$)$ & 16.5 & $2 \times 10^{18}$ & graded & 0.10 & graded \\
\hline $\mathrm{p}-\mathrm{Al}_{0.2} \mathrm{Ga}_{0.8} \mathrm{As}$ (cladding) & 300 & $5 \times 10^{17}$ & 135 & 0.17 & 3.50 \\
\hline p- $\mathrm{Al}_{0.2} \mathrm{Ga}_{0.8} \mathrm{As}$ (cladding) & 200 & $2 \times 10^{18}$ & 100 & 0.17 & 3.50 \\
\hline $\mathrm{p}-\mathrm{Al}_{0.2} \mathrm{Ga}_{0.8} \mathrm{As}$ (cladding) & 100 & $5 \times 10^{18}$ & 75 & 0.17 & 3.50 \\
\hline $\mathrm{i}-\mathrm{Al}_{0.5} \mathrm{Ga}_{0.5} \mathrm{As}$ (sublayer) & 3000 & $1 \times 10^{16}$ & 160 & 0.11 & 3.29 \\
\hline
\end{tabular}

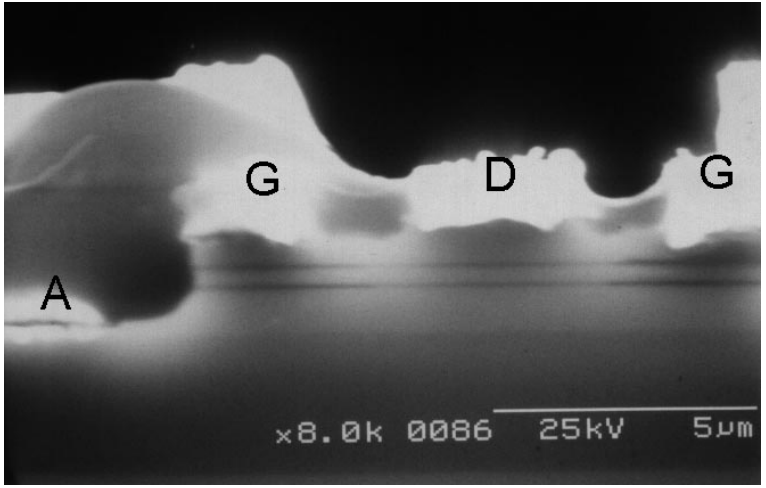

Fig. 2. Facet micrograph of a fabricated TAP detector. The dark lines are the oxidation layers, the white areas are metal contacts $(\mathrm{D}-$ detector contact; $\mathrm{G}$ - ground contact; $\mathrm{A}$-amplifier contact).

The $\mathrm{Al}_{x} \mathrm{Ga}_{1-x}$ As system converts from a direct semiconductor for $x<0.45$ to an indirect semiconductor for $x>0.45$. Our device contains both types of materials. The hole transport is not hindered by this transition. However, most electrons entering the $\mathrm{n}$-doped oxidation layers are transferred from the $\Gamma$ valley into the $X$ side valley of the conduction band. Due to the high $\mathrm{Al}$ mole fraction, a negligible number of electrons is still traveling in the $\Gamma$ valley. Thus, the lower band edge is used

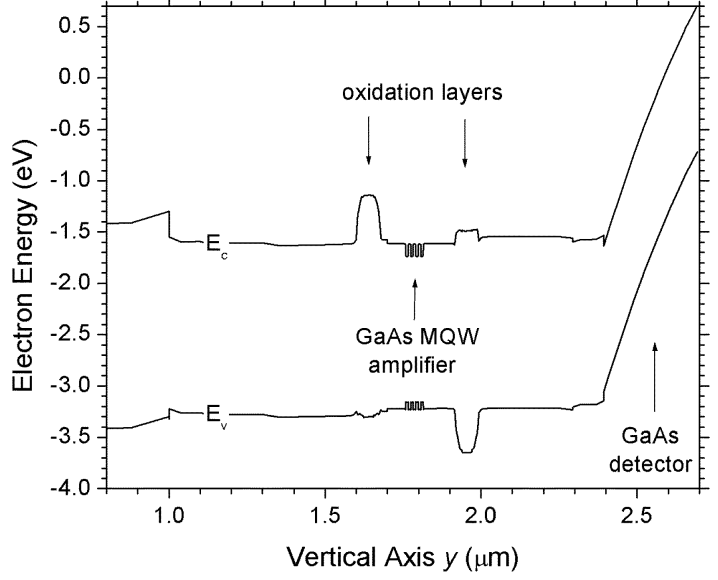

Fig. 3. Vertical band diagram in the center of the device $\left(E_{c}\right.$-conduction band edge; $E_{v}$-valence band edge)

in calculating the carrier transport across hetero-interfaces. The common band offset ratio of $\Delta E_{c} / \Delta E_{v}=60: 40$ is employed at all interfaces. Fig. 3 shows the band diagram at the vertical axis of the device. The top detector contact exhibits a Schottky barrier of $0.7 \mathrm{eV}$. The detector bias is $V_{D}=0 \mathrm{~V}$ in our investigation. Due to the grading and doping profile used, the valence 


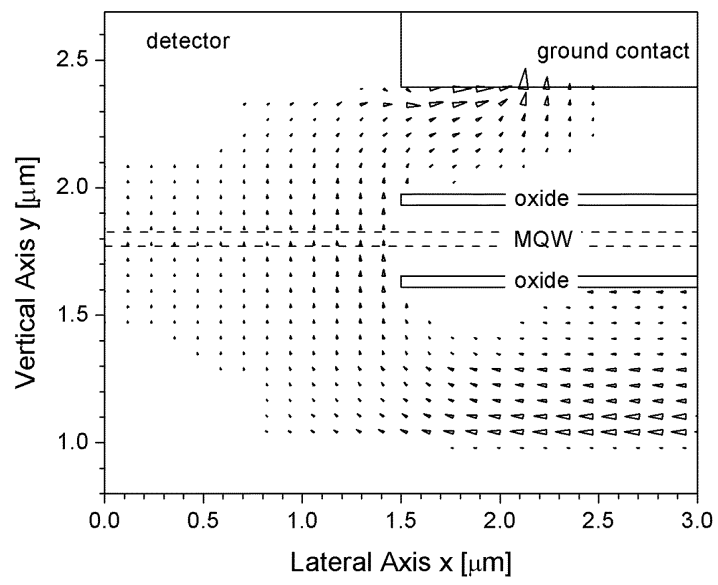

Fig. 4. Vector plot of the current distribution in the center of the device at $I_{A}=100 \mathrm{~mA}$ amplifier current (the arrows scale with the current density and disappear for less than $10 \%$ of the maximum). The left border marks the device symmetry plane.

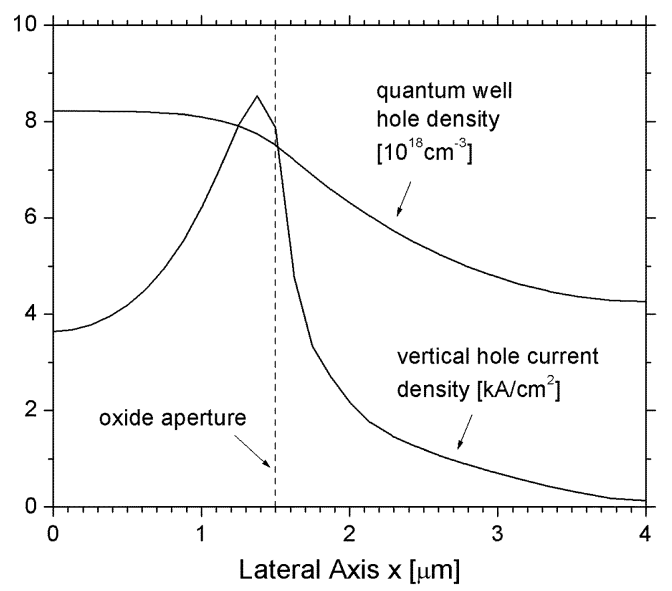

Fig. 5. Lateral profiles of the vertical hole current component and the hole density in the second quantum well at $100 \mathrm{~mA}$ amplifier current. The dashed line indicates the oxide aperture.

band edge of the oxidation layers is almost flat on the $p$-doped side and it hardly limits the hole injection into the MQW amplification region.

Fig. 4 gives a 2-D vector plot of the amplifier current distribution without light input. The MQW injection current is minimum in the center of the device. The lateral distribution of the hole current density in the amplification region is shown in Fig. 5 as well as the lateral hole density profile. Despite the current crowding caused by the oxide aperture layers, the hole distribution in the center of the device is almost uniform due to lateral carrier diffusion in the quantum wells. However, the same diffusion also causes significant lateral carrier leakage into the oxide region.

Without input light, more than $80 \%$ of the injected amplifier current is consumed by spontaneous emission of photons. The remaining current mainly feeds Auger recombination within the quantum wells (Auger coefficient $C=1.5 \times 10^{-30} \mathrm{~cm}^{6} \mathrm{~S}^{-1}$ ). A Shockley-Read-Hall (SRH) recombination lifetime of $100 \mathrm{~ns}$ is considered in our simulation, resulting in negligibly small defect recombination.

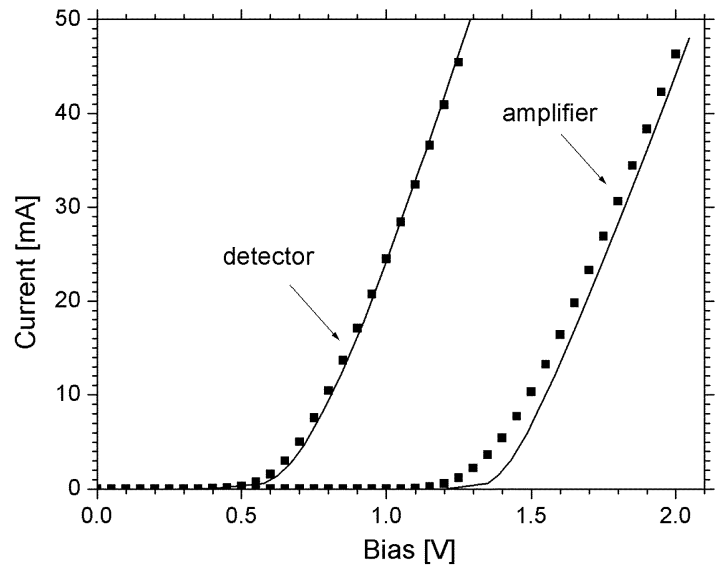

Fig. 6. Comparison of calculated (line) and measured (dots) current-voltage characteristics at forward bias $(L=600 \mu) \mathrm{m}$.

Current-voltage (IV) characteristics at forward bias are calculated for both the amplifier and the detector. Good agreement with measurements is obtained considering the ground contact resistivity of $1.8 \times 10^{-4} \Omega \cdot \mathrm{cm}^{2}$ (Fig. 6).

\section{SELF-HeAting}

Joule heating is the main heat source in our device. Most of the photons generated by spontaneous emission are either leaving the central region of the device or being absorbed in the GaAs ridge. Self-heating changes electrical and optical material properties and it may affect the device performance. The thermal conductivity values used in our thermal simulation consider the strong effect of alloy scattering in AlGaAs [7] as well as interface scattering in thin layers [8] (Table I). Outside the simulated device region, a lumped external thermal resistance of $50 \mathrm{~K} / \mathrm{W}$ is employed to represent the GaAs substrate and the heat sink.

The calculated internal temperature distribution is shown in Fig. 7. Due to the contact resistance, the peak temperature of $310 \mathrm{~K}$ occurs at the ground contact. The maximum temperature in amplifier and detector layers is 306 and $307 \mathrm{~K}$, respectively. The temperature rise outside the simulated device region is only $2 \mathrm{~K}$. Thus, self-heating seems to be relatively small in our device and it will be neglected in the following. Improvements of the $n$-contact resistance are expected with future devices, which will further reduce the heat power generated.

\section{GAIN AND ABSORPTION}

The conduction bands of the quantum wells are assumed parabolic and the nonparabolic valence bands are computed by the two-band $\vec{k} \cdot \overrightarrow{\mathbf{p}}$ method [9]. Based on this band structure, the optical gain is calculated using a free carrier model and including Lorentz broadening with $0.1 \mathrm{ps}$ carrier relaxation time [5]. The GaAs band gap shrinkage $\Delta E_{g}$ with higher carrier density $(n=p)$ is considered as [10]

$$
\Delta E_{\mathrm{g}}=-21 \mathrm{meV} \times\left(\frac{n}{10^{18} \mathrm{~cm}^{-3}}\right)^{\frac{1}{3}} .
$$

The resulting spectra of the average MQW gain are plotted in Fig. 8 for different amplifier currents. The wavelength of max- 


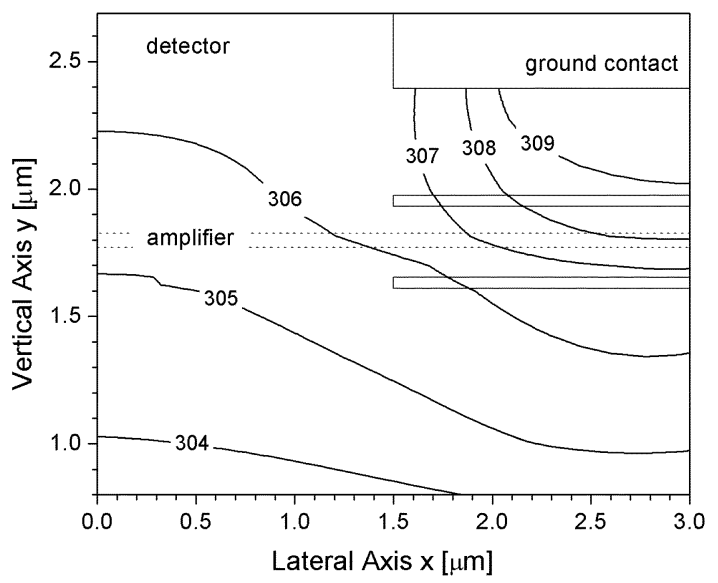

Fig. 7. Contour plot of the calculated temperature distribution at $100 \mathrm{~mA}$ amplifier current and $300 \mathrm{~K}$ ambient temperature.

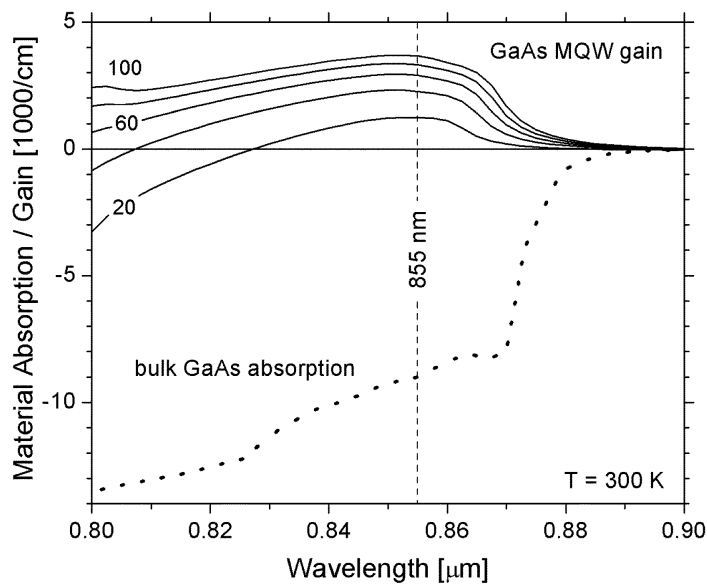

Fig. 8. Gain and absorption spectra for MQW and ridge, respectively, at room temperature. Gain spectra are calculated at 20,40,60, 80, and $100 \mathrm{~mA}$ amplifier current. The dots represent measured absorption data.

imum amplifier gain is about $855 \mathrm{~nm}$, which is in perfect agreement with our experimental observations.

Also shown in Fig. 8 is the measured band-to-band absorption spectrum of the detector with an absorption coefficient of $\alpha_{\operatorname{det}}=9000 \mathrm{~cm}^{-1}$ at $855 \mathrm{~nm}$ [11]. Detrimental waveguide absorption is caused by free carriers and is proportional to the local density of electrons $(n)$ and holes $(p)$ [12]

$$
\alpha_{\mathrm{f}}=3 \mathrm{~cm}^{-1} \times\left(\frac{n}{10^{18} \mathrm{~cm}^{-3}}\right)+7 \mathrm{~cm}^{-1} \times\left(\frac{p}{10^{18} \mathrm{~cm}^{-3}}\right) .
$$

At our maximum amplifier current of $100 \mathrm{~mA}$, the amplifier gain is only $g_{\mathrm{amp}}=3700 \mathrm{~cm}^{-1}$, far below the detector absorption. This discrepancy is enhanced by the difference in layer thickness. The four quantum wells give a total amplification layer thickness of $32 \mathrm{~nm}$, which is much smaller than the absorption layer thickness of $300 \mathrm{~nm}$. Thus, optical waveguide modes are preferred that are mainly confined to the MQW region.

\section{OPTICAL MODES}

The vertical profile of the refractive index is plotted in the bottom part of Fig. 9. Lateral mode confinement is provided by

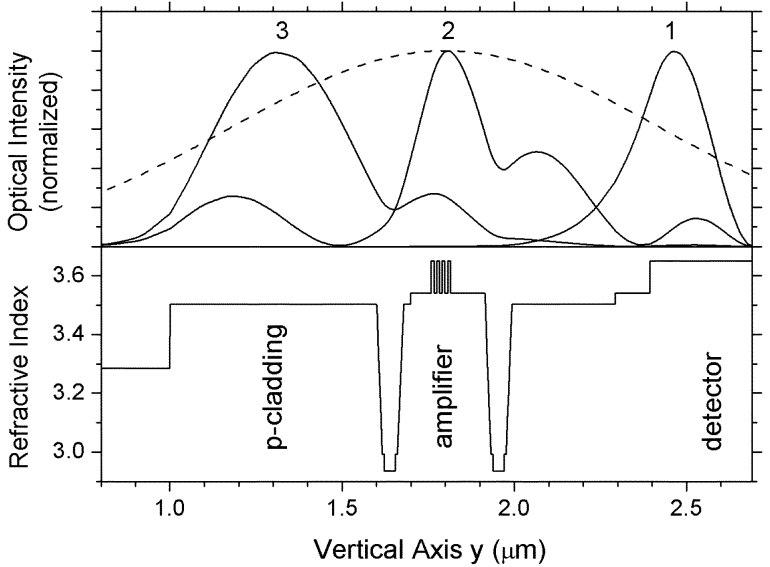

Fig. 9. Top: vertical intensity profile of the main waveguide modes (solid) and the input beam (dashed). Bottom: vertical refractive index profile.

TABLE II

Fiber COUPLing FACTORS $c_{\mathrm{f}}$ (Without REFLECTION) AND OPTICAL CONFINEMENT FACTORS $\Gamma_{\text {amp }}$ AND $\Gamma_{\text {det }}$ OF THE VERTICAL MODES IN Fig. 9 FOR AMPLIFICATION AND DETECTION LAYERS, RESPECTIVELY

\begin{tabular}{l|l|l|l}
\hline Mode & $c_{\mathrm{f}}$ & $\Gamma_{\mathrm{amp}}$ & $\Gamma_{\text {det }}$ \\
\hline 1 & 0.231 & 0.00021 & 0.63390 \\
\hline 2 & 0.165 & 0.07562 & 0.06412 \\
\hline 3 & 0.423 & 0.01187 & 0.00227 \\
\hline
\end{tabular}

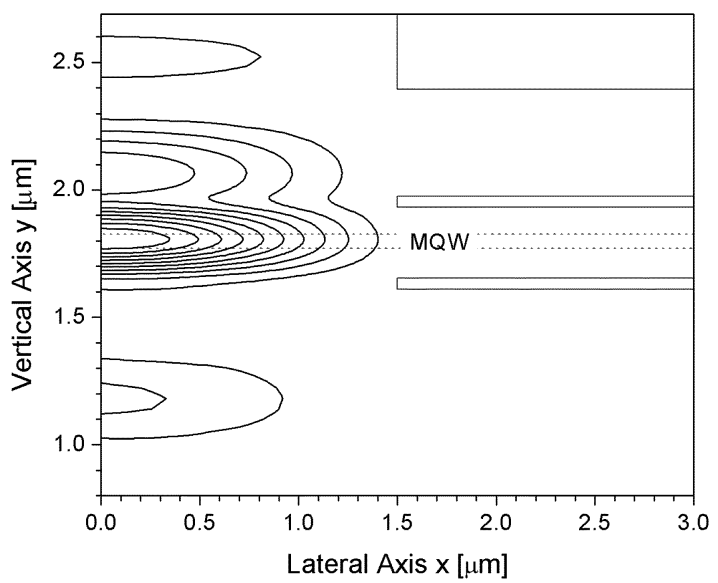

Fig. 10. 2-D intensity distribution of the amplifier mode.

the top ridge as well as by the oxide layers. A value of 1.7 is used for the refractive index of aluminum oxide. Vertical profiles of the three main waveguide modes are plotted as solid lines in the top part of Fig. 9. The intensity profile of the external light beam is given as a dashed line, considering light input from a perfectly aligned lensed fiber with $2.5 \mu \mathrm{m}$ spot size. Fiber coupling factors and optical confinement factors are given in Table II. Mode 1 is mainly located in the detector region, mode 2 in the amplifier region, and mode 3 does not show much overlap with any active layer. The amplifier mode 2 seems to be most desirable for our device; its 2-D contour is shown in Fig. 10. Without facet reflection, up to $82 \%$ of the input power is coupled into these three main waveguide modes. Most of the remaining 


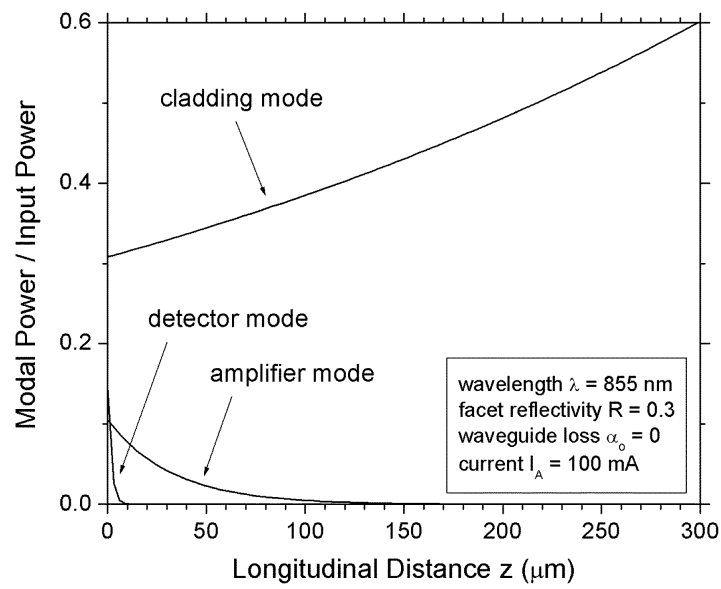

Fig. 11. Relative modal power versus travel distance for the three main modes.

light power is lost in unguided (leaky) optical modes. The total modal gain of each mode is given as

$$
g_{\mathrm{m}}=\Gamma_{\mathrm{amp}} g_{\mathrm{amp}}-\Gamma_{\mathrm{det}} \alpha_{\mathrm{det}}-\alpha_{\mathrm{o}}
$$

with the waveguide loss parameter $\alpha_{0}$, which includes free-carrier absorption and photon scattering losses, e.g., due to the oxide aperture roughness. For any given wavelength and current, the parameters $g_{\mathrm{amp}}$ and $\alpha_{\text {det }}$ can be extracted from Fig. 8 . The relative modal power in travel direction $z$ is given by

$$
\frac{P_{\mathrm{m}}}{P_{\mathrm{in}}}=(1-R) c_{\mathrm{f}} \exp \left(g_{\mathrm{m}} z\right)
$$

with the input light power $P_{\text {in }}$ and the facet power reflectance $R$. For $R=0.3$, the modal power is plotted in Fig. 11 with $100 \mathrm{~mA}$ amplifier current. The detector mode 1 is completely absorbed after a short travel distance. The amplifier mode 2 also suffers from net absorption. Only the cladding mode 3 exhibits positive gain. However, its small overlap with the detector limits the photocurrent.

\section{DETECTOR EFFICIENCY}

Based on the data above, we are now able to calculate the photocurrent for each mode [2]

$$
I_{\mathrm{m}}=\frac{q}{h \nu} P_{\mathrm{in}}(1-R) c_{\mathrm{f}} \Gamma_{\mathrm{det}} \alpha_{\mathrm{det}} \frac{\exp \left(g_{\mathrm{m}} L\right)-1}{g_{\mathrm{m}}}
$$

with the electron charge $q$ and the photon energy $h \nu$. Fig. 12 plots the modal photocurrents as function of the amplifier current for $268 \mu \mathrm{W}$ input power and $R=0.3$. As expected, the current from the detector mode is not affected by the amplification. Initially, it provides the strongest contribution to the total photocurrent, due to the mode confinement to the detector layer. The cladding mode contains the highest initial power and it experiences significant gain. Its photocurrent dominates at higher amplifier current, despite the small overlap with the detector. The amplifier mode 2 exhibits the strongest confinement to the amplifier region and it therefore contributes substantially to the

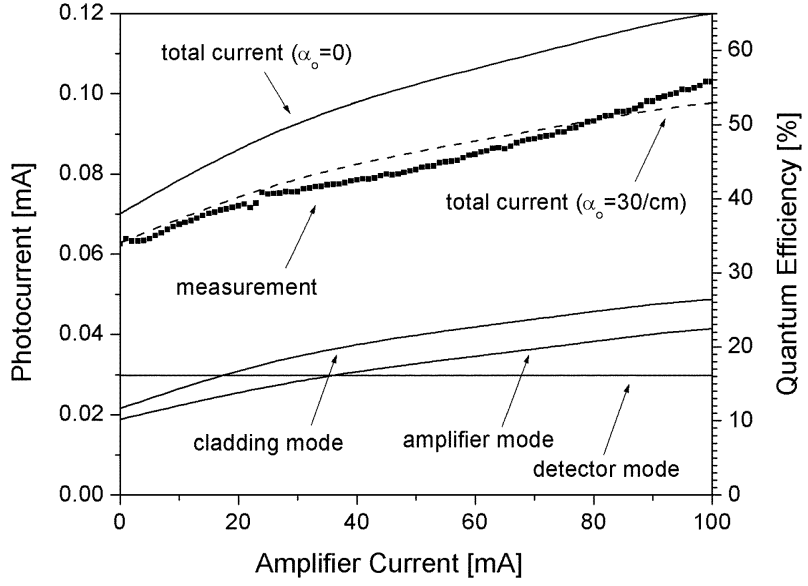

Fig. 12. Photocurrent and quantum efficiency versus amplifier current for 268 $\mu \mathrm{W}$ input power at $855 \mathrm{~nm}$ (lines-simulation; dots-measurement).

photocurrent enhancement. The sum of all three modal currents gives the total photocurrent in Fig. 12 (solid line), which is surprisingly close to the measurement, considering that no parameter fit was performed in our simulation thus far. We selected the measurement with the highest photocurrent, as obtained by optimum fiber alignment. Better agreement with this measurement can be achieved by assuming waveguide losses of $\alpha_{\mathrm{o}}=30 \mathrm{~cm}^{-1}$ in our simulation (dashed line in Fig. 12). Only a small part of this waveguide loss is attributed to free-carrier absorption $\left(\alpha_{\mathrm{f}}=6 \mathrm{~cm}^{-1}\right.$ for mode 2 at $\left.100 \mathrm{~mA}\right)$. Photon scattering seems to dominate $\alpha_{\mathrm{o}}$. However, this fit parameter also compensates for other uncertainties, such as the photocurrent from unguided light or inaccurate gain calculation.

The maximum measured photocurrent corresponds to only $56 \%$ quantum efficiency, much less than anticipated. Our goal was to surpass $100 \%$ quantum efficiency given by the photocurrent

$$
I_{\mathrm{ph}}^{100 \%}=\frac{q}{h \nu} P_{\mathrm{in}}
$$

which amounts to $0.185 \mathrm{~mA}$ in our case. The simulation reveals several reasons why this goal cannot be accomplished with our present device. A major reason is the imbalance of amplification and detection in our waveguide modes. Photons that are multiplied by stimulated emission stay within the same mode. Ideally, an equal number of photons should be generated and absorbed in each mode. This would give constant modal power along the device so that the photocurrent would continuously increase with the device length, without saturation. Within such a balanced mode, the quantum well gain should be as high as possible to achieve high photon throughput to the detector. The amplifier mode has the highest confinement factor $\Gamma_{\mathrm{amp}}$ and the best potential to reach this goal. However, it experiences too much absorption. The cladding mode exhibits little overlap with the detector region which results in constant optical power at low amplifier current of $I_{A}=30 \mathrm{~mA}$ (cf. Fig. 11, $\alpha_{\mathrm{o}}=0$ ). But it is not the preferred mode as its MQW gain is relatively small. It also diverts light power from the more productive amplifier mode. Finally, the detector mode only gives a constant photocurrent and it hardly contributes to the light amplification. 


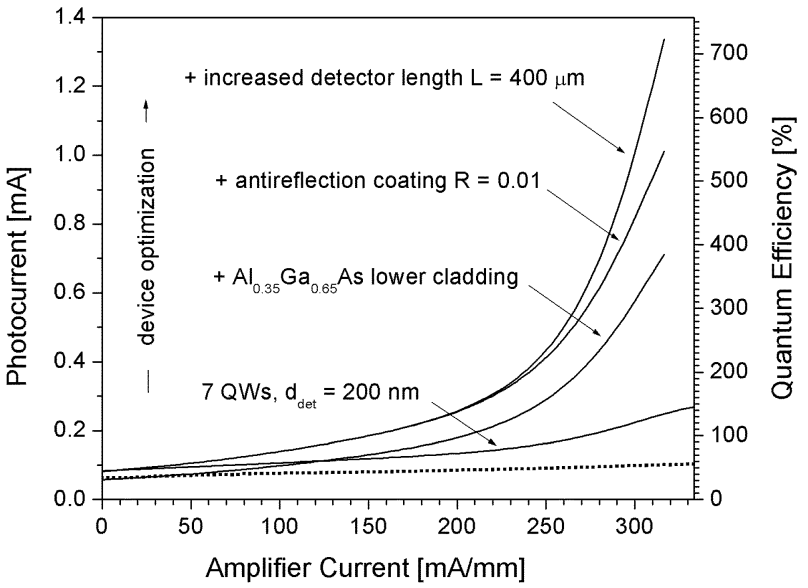

Fig. 13. Photocurrent and quantum efficiency versus amplifier current with optimized device design $\left(P_{\mathrm{in}}=268 \mu \mathrm{W}, \alpha_{\mathrm{o}}=30 \mathrm{~cm}^{-1}\right.$; lines-simulation; dots-original measurement)

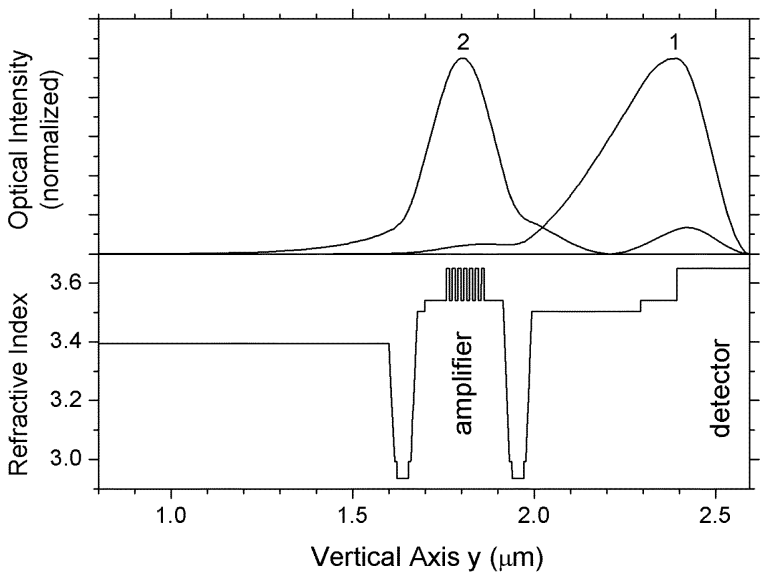

Fig. 14. Vertical profiles of refractive index (bottom) and optical modes (top) for the optimized device design.

\section{Device Optimization}

Following the analysis above, we now optimize the device design in order to better support the amplifier mode and to suppress the other two modes. First, the amplifier gain is increased by raising the number of quantum wells, while maintaining the total thickness of the amplifier waveguide. Seven quantum wells are found to be optimum, increasing the modal gain from $280 \mathrm{~cm}^{-1}$ to $400 \mathrm{~cm}^{-1}\left(I_{A}=100 \mathrm{~mA}\right)$. Second, the modal detector absorption needs to be lowered in order to match the modal amplifier gain. A reduced detector thickness of $200 \mathrm{~nm}$ accomplishes this balance for the amplifier mode. At this point, the TAP detector is still affected by three optical modes. The corresponding total photocurrent is shown in Fig. 13 with a maximum of $145 \%$ quantum efficiency.

The next step is the elimination of the cladding mode by using a uniform $\mathrm{Al}_{x} \mathrm{Ga}_{1-x} \mathrm{As}$ composition of $x=0.35$ below the oxide layer. The resulting profiles of the refractive index and of the two remaining optical modes are plotted in Fig. 14. The corresponding coupling and confinement factors are given in Table III. Both of the modes have less overlap with the detector region than in Table II. Up to $61 \%$ of the input power can now be coupled into the waveguide, compared to $82 \%$ with
TABLE III

Fiber COUPLING FACTORS $c_{\mathrm{f}}$ (WITHOUT REFLECTION) AND OPTICAL CONFINEMENT Factors $\Gamma_{\text {amp }}$ AND $\Gamma_{\text {det }}$ OF THE Two Modes IN Fig. 14 FOR AMPLIFICATION AND DETECTION LAYERS, RESPECTIVELY

\begin{tabular}{l|l|l|l}
\hline Mode & $c_{\mathrm{f}}$ & $\Gamma_{\mathrm{amp}}$ & $\Gamma_{\text {det }}$ \\
\hline 1 & 0.266 & 0.00744 & 0.29228 \\
\hline 2 & 0.342 & 0.19291 & 0.05629 \\
\hline
\end{tabular}

the original design. However, the fiber coupling factor of the highly productive amplifier mode 2 has more than doubled, which gives a much improved detector efficiency (Fig. 13). The amplifier mode is perfectly balanced at $95 \mathrm{~mA}$ amplifier current $(317 \mathrm{~mA} / \mathrm{mm})$, which is considered the upper limit of detector operation in order to avoid saturation effects. The maximum quantum efficiency at this current is $390 \%$. The detector mode contributes only $0.034 \mathrm{~mA}$ to the photocurrent.

Antireflection coating of the front facet $(R=0.01)$ and an increased device length $(L=400 \mu \mathrm{m})$ further enhance the quantum efficiency (Fig. 13). Due to microwave propagation loss, longer devices are expected to reduce the bandwidth below $40 \mathrm{GHz}$. [2] Overall, our device optimization promises more than $700 \%$ quantum efficiency, which represents an improvement of the original performance by more than one order of magnitude.

\section{SUMMARY}

We have evaluated device physics and performance of oxide-confined amplification photodetectors. The simulation is in good agreement with the experimental results. The poor quantum efficiency measured is confirmed by the simulation. Most of the input power is found to be coupled into inefficient waveguide modes with weak amplification effect. Device optimization is performed in order to enhance the amplification of the most efficient waveguide mode and to suppress all other modes. Simulation of the optimized device shows more than ten times improvement of the detection efficiency, which surpasses $700 \%$.

\section{REFERENCES}

[1] D. Lasaosa, Y. J. Chiu, J. Piprek, and J. E. Bowers, "Traveling-wave amplification photodetector (TAP detector)," in 13th Lasers and Electro-Optics Society Annu. Meeting. Piscataway: IEEE Press, 2000, pp. $260-261$.

[2] - "Modeling of traveling-wave amplification photodetectors," in Physics and Simulation of Optoelectronic Devices IX. Bellingham, WA: International Society of Optical Engineering, 2001.

[3] APSYS 4.3.3, Crosslight Software (2001). Available: http://www. crosslight.com. [Online]

[4] BeamPROP 5.0, RSoft Design Group (2001). Available: http://www. rsoftdesign.com. [Online]

[5] J. Piprek, Semiconductor Optoelectronic Devices-Introduction to Physics and Simulation. San Diego, CA: Academic, 2003.

[6] J. S. Blakemore, "Semiconducting and other major properties of gallium arsenide," J. Appl. Phys., vol. 53, pp. R123-R181, 1982.

[7] W. Nakwaski, "Thermal conductivity of binary, ternary and quaternary III-V compounds," J. Appl. Phys., vol. 64, pp. 159-166, 1988.

[8] J. Piprek, T. Troger, B. Schroter, J. Kolodzey, and C. S. Ih, "Thermal conductivity reduction in GaAs-AlAs distributed Bragg reflectors," IEEE Photon. Technol. Lett., vol. 10, pp. 81-83, Jan. 1998.

[9] S. L. Chuang, Physics of Optoelectronic Devices. New York: Wiley, 1995.

[10] R. Zimmermann, Many-Particle Theory of Highly Excited Semiconductors. Leipzig, Germany: Teubner Verlagsgesellschaft, 1988. 
[11] Handbook of Optical Constants of Solids, E. D. Palik, Ed., Academic, San Diego, 1998.

[12] Handbook of Semiconductor Lasers and Photonic Integrated Circuits, Y. Suematsu and A. R. Adams, Eds., Chapman \& Hall, London, U.K., 1994.

Joachim Piprek (M'94-SM'98) received the Ph.D. degree in solid-state physics from Humboldt University, Berlin, Germany, in 1986.

$\mathrm{He}$ is currently an Adjunct Associate Professor at the University of California, Santa Barbara. He has worked in the industry and academia on design and analysis of optoelectronic devices and has given more than 40 invited talks, seminars, and short courses in the field. He has authored more than 50 papers in refereed journals, two book chapters, and a book entitled, Semiconductor Optoelectronic Devices-Introduction to Physics and Simulation (New York: Academic, 2003).

Prof. Piprek chairs the SPIE Symposium on Semiconductor Optoelectronic Devices for Lightwave Communication as well as the IEEE/LEOS International Conference on Numerical Simulation of Semiconductor Optoelectronic Devices.

Daniel Lasaosa received the B.E. degree from the Public University of Navarra, Navarra, Spain, and the M.E. degree from the National Technical Institute, Grenoble, France, both in 1995, and the M.S. degree in 1997 from the University of California, Santa Barbara, where he is currently pursuing the $\mathrm{Ph} . \mathrm{D}$. degree.

His research interests include traveling-wave photodetectors with optical amplification.
Donato Pasquariello (S'00-A'01) received the Ph.D. degree from Uppsala University, Uppsala, Sweden, in 2001

At the Ångström Laboratory, Uppsala University, he worked on micro-optoelectro-mechanical-systems and wafer bonding. He joined Prof. Bowers' group at he University of California, Santa Barbara in the summer of 2001. Currently, his research is focused on InP-based traveling-wave photodetectors.

John E. Bowers (S'78-M'81-SM'85-F'93) received the M.S. and Ph.D. degrees from Stanford University, Stanford, CA, in 1978 and 1981, respectively.

He is Director of the Multidisciplinary Optical Switching Technology Center (MOST) and a Professor in the Department of Electrical Engineering, University of California, Santa Barbara (UCSB). He is cofounder of the Center for Entrepreneurship and Engineering Management, a founder of Terabit Technology and Calient Networks and serves on the Board of Directors of Calient Networks. He had worked for AT\&T Bell Laboratories and Honeywell before joining UCSB. He has published six book chapters, over 250 journal papers, over 250 conference papers, and holds 26 patents. His research interests are primarily concerned with optoelectronic devices and optical networking.

Prof. Bowers is a Fellow of the OSA and the American Physical Society. He is a recipient of the IEEE LEOS William Streifer Award. 